\title{
Enhancing Navigation in Difficult Environments with Low-Cost, Dual-Frequency GNSS PPP and MEMS IMU
}

\author{
Sudha Vana and Sunil Bisnath
}

\begin{abstract}
The Global Navigation Satellite System (GNSS) Precise Point Positioning (PPP) technology benefits from not needing local ground infrastructure such as reference stations and accuracy attained is at the decimetre-level, which approaches real-time kinematic (RTK) performance. However, due to its long position solution initialization period and complete dependence on the receiver measurements, PPP finds limited utility. The emergence of low-cost, micro-electro-mechanical sensor (MEMS) inertial measurement units (IMUs) has prompted research in integrated navigation solutions with the PPP processing technique. This sensor fusion aids to achieve continuous positioning and navigation solution availability when there are insufficient numbers of navigation satellites visible. In the past, research has been conducted to integrate high-end (geodetic) GNSS receivers with PPP processing and MEMS IMUs, or low-cost, single-frequency GNSS receivers with point positioning processing and MEMS IMUs. The objective of this research is to investigate and analyze position solution availability and continuity by integrating low-cost, dual-frequency GNSS receivers using PPP processing with the latest low-cost, MEMS IMUs to offer a complete, low-cost navigation solution that will enable continuously available positioning and navigation solutions, even in obstructed environments. The horizontal accuracy of the developed low-cost, dual-frequency GNSS PPP with MEMS IMU integrated algorithm is approximately $20 \mathrm{~cm}$. During half a minute of simulated GNSS signal outage, the integrated solution offers $40 \mathrm{~cm}$ horizontal accuracy. A low-cost, dual-frequency GNSS receiver PPP solution integrated with a MEMS IMU forms a unique combination of a total low-cost solution, that will open a significant new market window for modern-day applications such as autonomous vehicles, drones and augmented reality.
\end{abstract}

\section{Keywords}

Global Navigation Satellite System (GNSS) - Global positioning system (GPS) • Inertial measurement unit (IMU) · Low-cost navigation • Precise Point Positioning (PPP) • PPP + IMU

Presented at IUGG 2019 General Assembly.

S. Vana $(\bowtie) \cdot$ S. Bisnath

Department of Earth and Space Science and Engineering, York

University, Toronto, ON, Canada

e-mail: sudhav@yorku.ca

\section{Introduction}

Obtaining continuous and accurate navigation solutions in any environment is a challenge because GNSS signals are obstructed in environments such as downtowns, tunnels or areas covered with foliage. Integrating the GNSS sensor with another self-contained navigation sensor such as an Inertial Measurement Unit (IMU) becomes necessary in such cases. 
The advent of IMUs based on micro-electro-mechanical sensors (MEMS) has brought a whole new market of lowcost IMU sensors. MEMS IMU sensors are cheaper by price but also come with some significant in-built errors such as bias, noise and scale factor (Abd Rabbou and ElRabbany 2015). Shin et al. (2005), Abdel-Hamid et al. (2006), Scherzinger (2000) and many other researchers have all performed integrated navigation of GNSS and MEMS IMU by applying Differential GPS (DGPS) or Real-Time Kinematic (RTK) techniques to improve continuity and accuracy of the navigation solution in the event of GNSS signal outage. Precise Point Positioning (PPP) is an augmentation technique that does not require a local reference station unlike the RTK technique (Zumberge et al. 1997). Precise orbit and clock information is broadcast via satellites or the Internet to the user. The performance accuracy achieved is decimetre- to centimetre-level. The initial convergence time of the PPP technique is minutes to the decimetre-level, which is one of its major drawbacks. PPP is a widely used technique for applications such as marine mapping, crustal deformation, airborne mapping, precision agriculture and construction applications (Seepersad 2012; Aggrey 2015; Aggrey and Bisnath 2017). PPP can be further augmented to reduce convergence period by applying satellite phase biases to obtain integer ambiguities and a priori atmospheric refraction information (Lannes and Prieur 2013; Teunissen and Khodabandeh 2015). This enables a stand-alone userreceiver to achieve RTK-like performance with a shorter convergence period, while limiting dependency on external infrastructure.

In the recent past, researchers have started applying the PPP technique to perform GNSS and MEMS IMU integration which has offered promising outcomes. Abd Rabbou and El-Rabbany (2015) experimented with GPS-PPP integration using a high-end GPS sensor and a MEMS IMU. The study showed decimetre-level accuracy with no GPS signal outages and during a $60 \mathrm{~s}$ of signal outage, sub-metre-level accuracies were demonstrated. Liu et al. (2018) conducted a study on integrating a low-cost, single-frequency (SF) GNSS with a MEMS IMU and were able to achieve centimetre- to decimetre-level accuracy with no GNSS signal loss. During a GNSS signal loss of $3 \mathrm{~s}$, the solution performed at the metre level.

The motivation of this research is to assess and analyze the performance of the recently emerging low-cost, dualfrequency (DF) GNSS receivers in the market integrated with a relatively low-cost MEMS IMU. The research questions addressed are: (1) What is the accuracy performance of a low-cost DF GNSS PPP receiver integrated with a MEMS IMU? And (2) What is the accuracy performance of a lowcost DF GNSS PPP receiver integrated with a MEMS IMU during a $30 \mathrm{~s}$ GNSS signal outage?
Modern-day applications such as low-cost vehicle autonomy, augmented reality, and pedestrian dead reckoning demand decimetre-level accuracy with low-cost sensors. Therefore, integrated, low-cost, DF GNSS PPP with MEMS IMU has the potential to offer accurate, continuous and precise navigation solution for the next generation of applications which is the objective of this research.

\section{Inertial Navigation System}

The raw measurements from an IMU, specific force and turn rates, are converted into position, velocity, and attitude using the IMU mechanization process. Known position, velocity and attitude are also inputs to the mechanization. The equation for accelerometer and gyro measurements with errors is as given below in Eqs. (1) and (2) (Farrell 2008).

$$
\begin{gathered}
\tilde{f}^{a}=\left(I-\delta S F_{a}\right)\left(f^{a}-\delta b_{a}-\delta n l_{a}-v_{a}\right) \\
\tilde{\omega}_{i p}^{g}=\left(I-\delta S F_{g}\right)\left(\omega_{i p}^{g}-\delta b_{g}-\delta k_{g}-v_{g}\right)
\end{gathered}
$$

$\tilde{f}^{a}$ is the actual accelerometer measurement that accounts for all measurement errors, $\delta S F_{a}$ is the accelerometer scale factor error, $\delta b_{a}$ is the accelerometer bias, $\delta n l_{a}$ is the accelerometer nonlinearity error, $v_{a}$ is the measurement noise. $\sim_{\omega}^{g}$ is the actual gyro measurement accounting for all measurement errors, $\delta S F_{g}$ is gyro scale factor, $\delta b_{g}$ is gyro bias, $\delta k_{g}$ represents gyro g-sensitivity, $v_{g}$ is measurement noise. $f^{a}$ and $\omega_{i p}^{g}$ are obtained in body-frame. After error compensations are made position and velocity can be calculated using IMU mechanization equations.

There are four main steps to the mechanization process: (1) Attitude update using the turn rates from gyroscopes; (2) reference frame conversion of specific force from body to the intended reference frame; (3) velocity update; and (4) position update.

Figure 1 depicts the IMU mechanization process. The details of mechanization in all the reference frames is explained in Groves (2013). For this research, the EarthCentred-Earth-Fixed (ECEF) frame of mechanization is used because the range measurements from the GNSS satellites can be used directly in the estimation process. In the Fig. 1, specific force $\boldsymbol{f}^{\boldsymbol{b}}$ from accelerometer and the angular rate $\boldsymbol{\omega}_{\boldsymbol{i b}}^{\boldsymbol{b}}$ from gyroscope are output in body frame. They are converted into the ECEF frame using direction cosine matrices or quaternions. After gravity compensation and Coriolis correction, along with the initial position, velocity and attitude estimates, time integration gives the current position, velocity and attitude. 


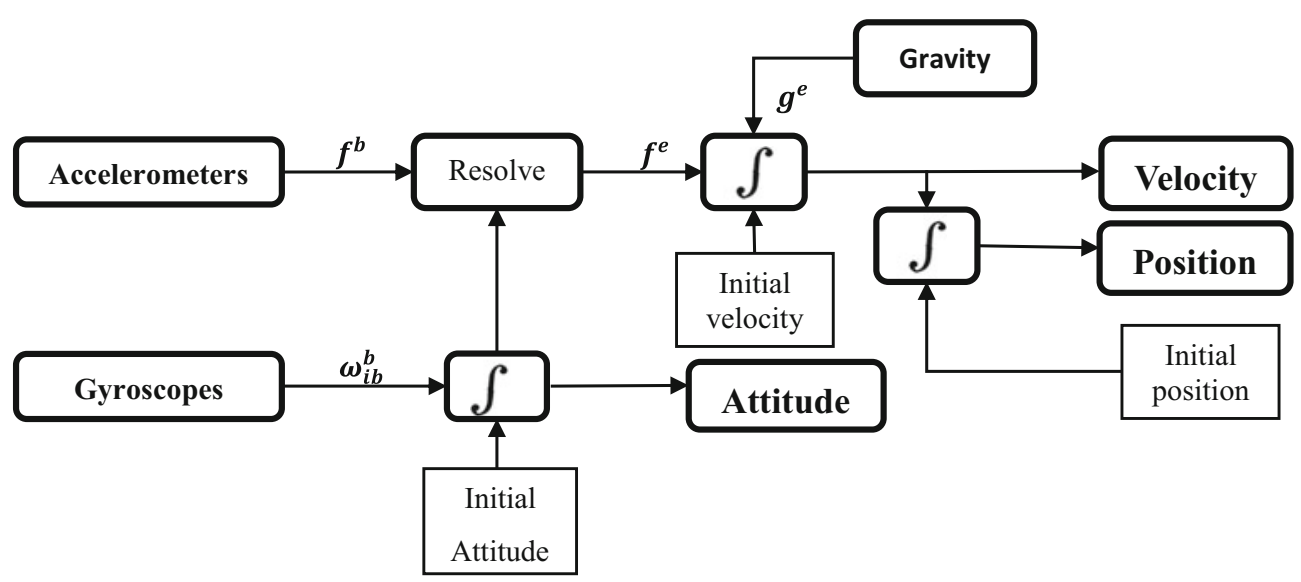

Fig. 1 Block diagram of IMU mechanization process (after Titterton et al. 2004)

Inputs to IMU mechanization are specific force $f^{b}$ and turn rates $\omega_{i b}^{b}$. Mechanization process including equations are described in detail in (Farrell 2008).

\section{GNSS PPP/INS Tightly Coupled Kalman Filter}

In this research, a tightly-coupled Extended Kalman Filter (EKF) is used to fuse the GNSS and IMU measurements. In a tightly-coupled integration architecture, raw measurements from the sensors are used, which enables continuous navigation during a GNSS signal outage. The typical error budget for GNSS PPP is listed in Table 1.

The inputs to the complementary Kalman filter are (1) code and phase measurements from a low-cost DF GNSS receiver corrected for atmosphere, relativistic errors and clock and orbit errors using the precise PPP corrections, and (2) predicted code and phase measurements that are formed using the IMU position and velocity with the satellite position and velocity. For this research work, the ionospherefree (IF) model is used to avoid estimation of the ionosphere, which simplifies the number of states to be estimated.

Table 1 PPP Error budget (Choy 2018)

\begin{tabular}{l|l}
\hline Error source & Error $(\mathrm{m})$ \\
\hline Ionosphere delay & $10-20$ \\
\hline Troposphere delay & $1-10$ \\
\hline Relativistic & 10 \\
\hline Multipath & 1.0 \\
\hline Receiver measurement noise & $0.1-0.7$ \\
\hline SV orbit/clock & $\sim 0.01-0.1$ \\
\hline Satellite phase centre variation & $0.05-1$ \\
\hline Solid earth tide & 0.2 \\
\hline Ocean loading & 0.05 \\
\hline Phase wind-up (ionosphere-free) & 0.1 \\
\hline Receiver phase centre variation & $0.001-0.01$ \\
\hline
\end{tabular}

The ambiguities estimated are float only. The mathematical model for IF PPP can be written as (Parkinson and Spilker 1996):

$$
\begin{gathered}
P=\rho+c\left(d t_{r}-d t^{s}\right)+T+c\left(B_{p}^{r}-B_{p}^{s}\right)+e_{P} \\
\varphi=\rho+c\left(d t_{r}-d t^{s}\right)+T+c\left(B_{\varphi}^{r}-B_{\varphi}^{s}\right)+N \lambda+e_{\varphi}
\end{gathered}
$$

In Eqs. (3) and (4), $d t_{r}$ and $d t^{s}$ are the receiver clock error and satellite clock errors respectively, $\mathrm{T}$ is the tropospheric delay, $B_{p}^{r}$ and $B_{p}^{s}$ are the code bias for receiver and satellite, $B_{\varphi}^{r}$ and $B_{\varphi}^{s}$ are the phase bias for receiver and satellite, $e_{P}$ and $e_{\varphi}$ are the unmodelled errors in pseudorange and carrier phase measurements, and $N \lambda$ is the ambiguity term between the receiver and satellite on phase measurements.

Figure 2 provides the representation of the EKF integration of the GNSS-PPP and IMU.

In Fig. 2, $f_{b}, w_{b}$ are the IMU specific force and turn rate measurements. These measurements are converted into position $P_{I M U}$, velocity $V_{I M U}$ and attitude $A_{I M U}$ from a known position, velocity and attitude by applying IMU mechanization process. Predicted $\rho_{I M U}, \varphi_{I M U}$ are constructed by using the satellite position and velocity, which are corrected by applying the precise orbit and clock corrections. DF code and phase measurements $\rho_{G N S S}, \varphi_{G N S S}$ are corrected for typical errors such as the errors mentioned in Table 1. The estimated output from the EKF are the error in IMU position $\delta r^{n}$, velocity $\delta v^{n}$ attitude $\delta \varepsilon^{n}$ and biases $b_{g}$ and $b_{a}$. $P_{I M U}^{e}, V_{I M U}^{e}$ and $A_{I M U}^{e}$ give the final IMU position, velocity and attitude.

The state vector consists of the navigation states, IMU states, and the GNSS only states. Navigation states include position error, velocity error and attitude error. While the inertial states consist of accelerometer and gyroscope biases. The GNSS states estimated are: GNSS receiver clock, as well 


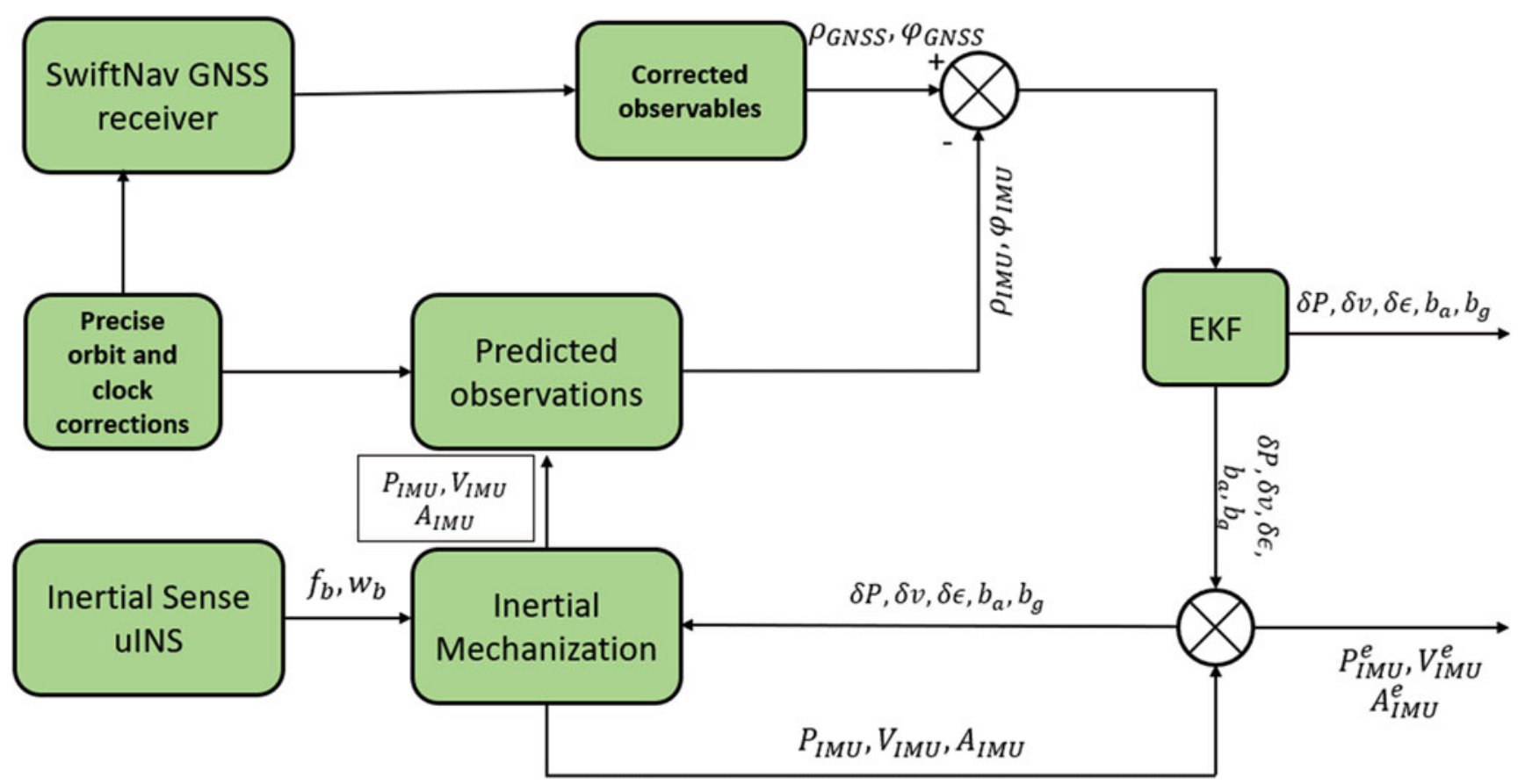

Fig. 2 Block diagram depicting PPP-INS tightly coupled integration

as drift and the float ambiguity terms. The state vector is represented mathematically in Eq. (5) (Jekeli 2012; Groves 2013, p. 201; Abd Rabbou and El-Rabbany 2015).

$$
\delta x=\left[\begin{array}{llllllllllll}
\delta P & \delta v & \delta \varepsilon & \delta t_{c} & \delta \dot{t}_{c} & d_{\text {tropo }} & b_{a} & b_{g} & N_{1} & N_{2} & \ldots & N_{n}
\end{array}\right]
$$

In the Eq. (5), $\delta P$ is the $3 \mathrm{D}$ position error, $\delta v$ is the $3 \mathrm{D}$ velocity error, $\delta \varepsilon$ is the attitude error, $\delta t_{c}$ GNSS receiver clock error, $\delta \dot{t}_{c}$ is GNSS receiver clock drift error, $d_{\text {tropo }}$ is the troposphere wet delay, $b_{a}$ and $b_{g}$ are accelerometer and gyroscope bias and $N_{i}$ float ambiguity of satellite i.

In continuous time, the transition matrix is given by Groves (2013).

$$
\begin{gathered}
F(t)=\left[\begin{array}{ccccc}
0_{3 \times 3} & I_{3 \times 3} & 0_{3 \times 3} & 0_{3 \times 3} & 0_{3 \times 3} \\
F_{23}^{e} & -2 \Omega_{i e}^{e} & F_{21}^{e} & C_{b}^{e} & 0_{3 \times 3} \\
0_{3 \times 3} & 0_{3 \times 3} & -\Omega_{i e}^{e} & 0_{3 \times 3} & C_{b}^{e} \\
0_{3 \times 3} & 0_{3 \times 3} & 0_{3 \times 3} & 0_{3 \times 3} & 0_{3 \times 3} \\
0_{3 \times 3} & 0_{3 \times 3} & 0_{3 \times 3} & 0_{3 \times 3} & 0_{3 \times 3}
\end{array}\right] \\
F_{21}^{e}=\left[-\left(C_{b}^{e} f_{i b}^{b}\right) \boldsymbol{X}\right] \quad F_{23}^{e}=-\frac{2 \widehat{\gamma_{l b}^{e}}}{r_{e S}^{e}\left(\widehat{L_{b}}\right)} \frac{\hat{r}_{e b}^{e} T}{\left|\hat{r}_{e b}^{e}\right|}
\end{gathered}
$$

Terms $F_{21}^{e}$ and $F_{23}^{e}$ are explained in detail in Groves (2013)

The measurement vector is the difference between corrected GNSS measurements, pseudorange, carrier phase and predicted IMU measurements. The measurement vector is given in Eq. (6)

$$
z=\left[\begin{array}{c}
\rho^{i}{ }_{G N S S}-\rho_{I N S}^{i} \\
\varphi^{i}{ }_{G N S S}-\varphi^{i}{ }_{I N S} \\
\vdots
\end{array}\right]
$$

In Eq. (6), $\rho^{i}$ is the pseudorange of the satellite $\mathrm{i}$ and $\varphi^{i}$ is the carrier phase measurement corresponding to satellite i. The design matrix will encompass the partial derivatives to the state terms related to GNSS. The other terms become zero.

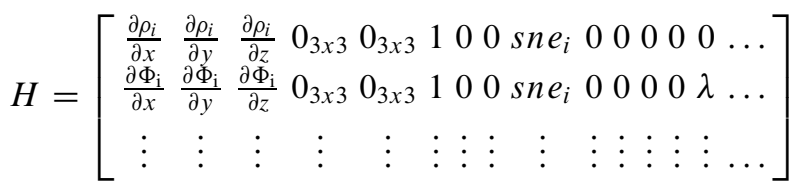

In Eq. (7), sne $e_{i}=\frac{1}{\sin (\text { elevation })}$ is the mapping function for the troposphere wet delay component. $\lambda$ is a partial derivative entry for the ambiguity terms $N \lambda$ which is wavelength corresponding to ambiguity $\mathrm{N}$.

\section{$4 \quad$ Field Test and Results}

To test and evaluate the tightly-coupled EKF, kinematic data were collected at the York University main campus in Toronto, Canada. A low-cost, dual-frequency receiver, 
Piksi-multi by Swift Navigation, and low-cost MEMS IMU, Inertial sense $\mu$ INS, were used. The Piksi is a multiconstellation, dual-frequency receiver that can offer $0.75 \mathrm{~m}$ accuracy in horizontal (CEP 50 in SBAS mode) (SwiftNav 2012). The Piksi is also capable of offering RTK like cm-level solutions with fast convergence with horizontal accuracy of $1 \mathrm{~cm}$. The inertial Sense $\mu$ INS consists of a SF uBlox GNSS receiver, magnetometer, barometer, and IMU. The MEMS IMU onboard $\mu$ INS is comparable to a tactical grade IMU based on the specifications (Inertial Sense 2013). The specifications of the InertialSense uINS is detailed in Table 2. As per the categorization of IMUs given in (Vector Nav Library 2008), uINS can be categorized as a tacticalgrade IMU from the specifications given in Table 2. The antenna used in the experiment is a geodetic grade antenna by SwiftNav. Since, a geodetic grade antenna was used in the setup, the quality of the measurements were better than the ones acquired using a low-cost antenna such as a patch antenna.

Both the GNSS and IMU sensors were placed beside each other in the car trunk. The geodetic grade antenna was installed on the car roof. The data logged consisted of a multi-constellation carrier phase and pseudorange informa-
Table 2 Specifications of uINS MEMS IMU

\begin{tabular}{l|l|l}
\hline Sensor & IMU-Gyros & IMU-Accels \\
\hline Operating range & $\pm 2,000^{\circ} / \mathrm{s}$ & $\pm 16 \mathrm{~g}$ \\
\hline Bias repeatability & $<0.2^{\circ} / \mathrm{s}$ & $<5 \mathrm{mg}$ \\
\hline In-run bias stability & $<10^{\circ} / \mathrm{h}$ & $<40 \mu \mathrm{g}$ \\
\hline Random walk & $0.15^{\circ} / \sqrt{h}$ & $0.07 \mathrm{~m} / \mathrm{s} / \sqrt{h}$ \\
\hline Non-linearity & $<0.1 \% \mathrm{FS}$ & $<0.5 \% \mathrm{FS}$ \\
\hline Noise density & $0.01^{\circ} / \mathrm{s} / \sqrt{\mathrm{Hz}}$ & $300 \mu \mathrm{g} / \sqrt{\mathrm{Hz}}$ \\
\hline
\end{tabular}

tion. Thus, collected raw observables were then processed using the York PPP + IMU algorithm for validation.

Figure 3 represents the track of data collected at a parking lot near the York University Campus in Toronto, Canada. The data were collected on October 12, 2019, DOY 168 for a period of $24 \mathrm{~min}$.

Logging data rate of GNSS observables was set to $5 \mathrm{~Hz}$ and the IMU data rate was set to $100 \mathrm{~Hz}$. Novatel's Waypoint software was used to post-process the measurements in RTK mode for the same data used as the reference. The processing parameters used for the data are summarized in Table 3.

Figure 4 represents a plot of GNSS satellites available during the span of data collection and corresponding position dilution of precision (PDOP). The average number of satel-

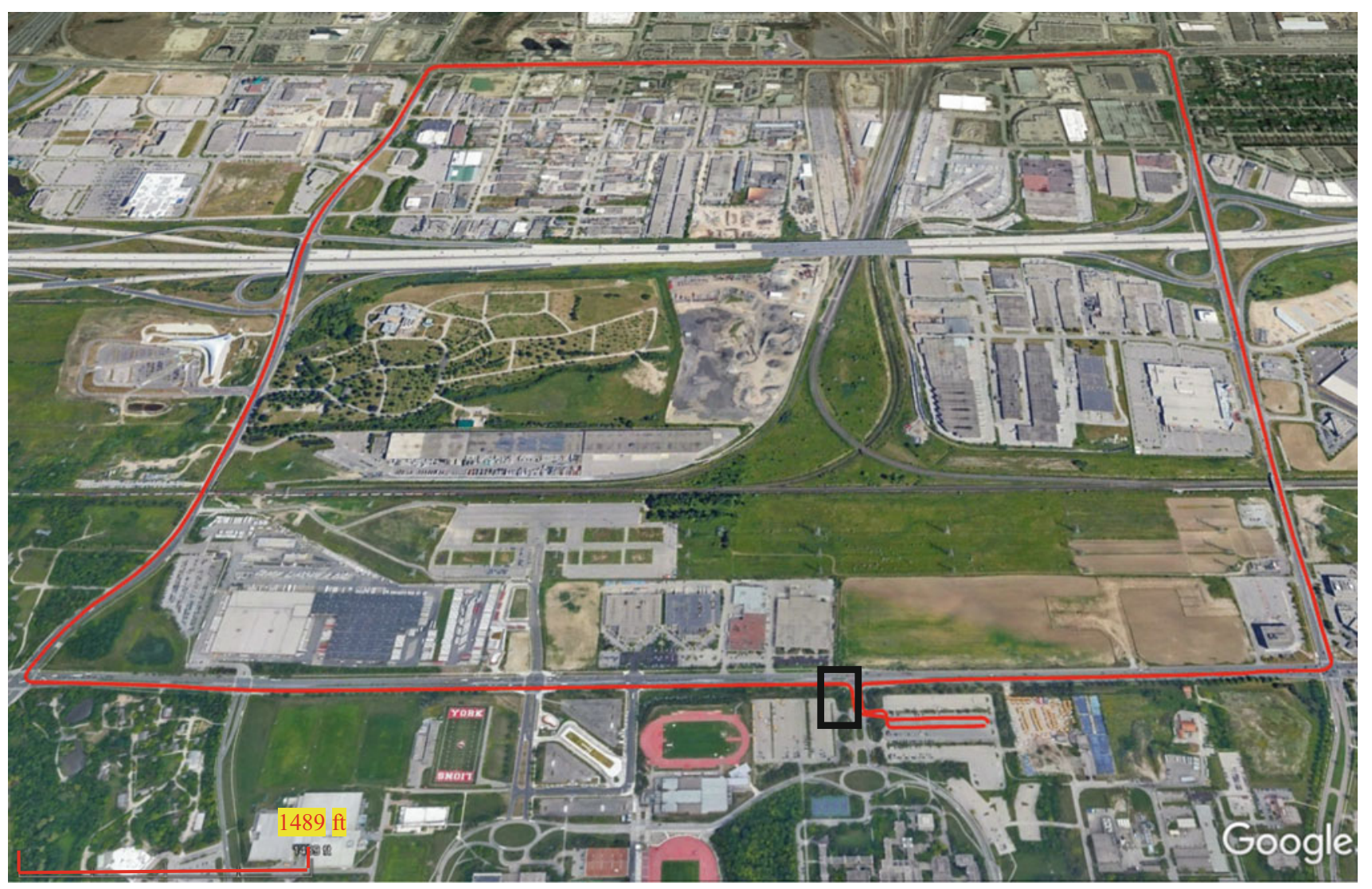

Fig. 3 Track of data collected around York University, Toronto, Canada 
Table 3 Processing parameters used for PPP + IMU TC algorithm

\begin{tabular}{l|l}
\hline Constellations processed & GPS, GLONASS, Galileo, BeiDou \\
\hline Elevation mask & $10^{\circ}$ \\
\hline Processing type & Iono-free combination \\
\hline $\begin{array}{l}\text { Satellite orbits and } \\
\text { clocks }\end{array}$ & $\begin{array}{l}\text { CNES products (http://www.ppp-wizard. } \\
\text { net/products/REAL_TIME/) }\end{array}$ \\
\hline $\begin{array}{l}\text { Observation processing } \\
\text { mode }\end{array}$ & Dual-frequency, kinematic processing \\
\hline Data format & RINEX 3.x
\end{tabular}

lites is 15 and the mean PDOP is 1.7. It is clear from Fig. 4 that the number of BeiDou satellites is much less compared to other constellations as the data were collected in North American region.

Figure 5 is a plot of horizontal and vertical error of the GNSS PPP and IMU solution when compared to the Novatel's Waypoint reference solution. The highlighted black box in the Fig. 3 is area where there are many trees and a signal outage took place. This can be seen in Fig. 5 as a jump in the position solution at $1,200 \mathrm{~s}$. The rms error of the solution is $23 \mathrm{~cm}$ in the horizontal direction and $33 \mathrm{~cm}$ in the vertical direction. The rms was calculated after the solution reaches convergence time which is $400 \mathrm{~s}$ and before signal outage due to trees happens at 1,200 s. The decimetrelevel performance of the algorithm makes it appropriate and suitable for the applications that require decimetre-level accuracy in positioning for a lower price.

Given the number of states that are estimated for the purpose of navigation from Eq. (5), at least five satellite raw observables are necessary to compute the user position. The evaluation of the performance of EKF during GNSS outage was done by simulating a GNSS signal outage for $30 \mathrm{~s}$ in the track. During the $30 \mathrm{~s}$ of simulated GNSS signal outage, the algorithm was tested with only four GNSS satellites available. Figure 6 is the horizontal error when compared to the reference solution during the GNSS signal outage of $30 \mathrm{~s}$. The blue solution is an error comparison with no outage while red-coloured error plot corresponds to the PPP + IMU performance during a $30 \mathrm{~s}$ outage. The simulated outage period is highlighted in black between 440 and $470 \mathrm{~s}$ in Fig. 6.
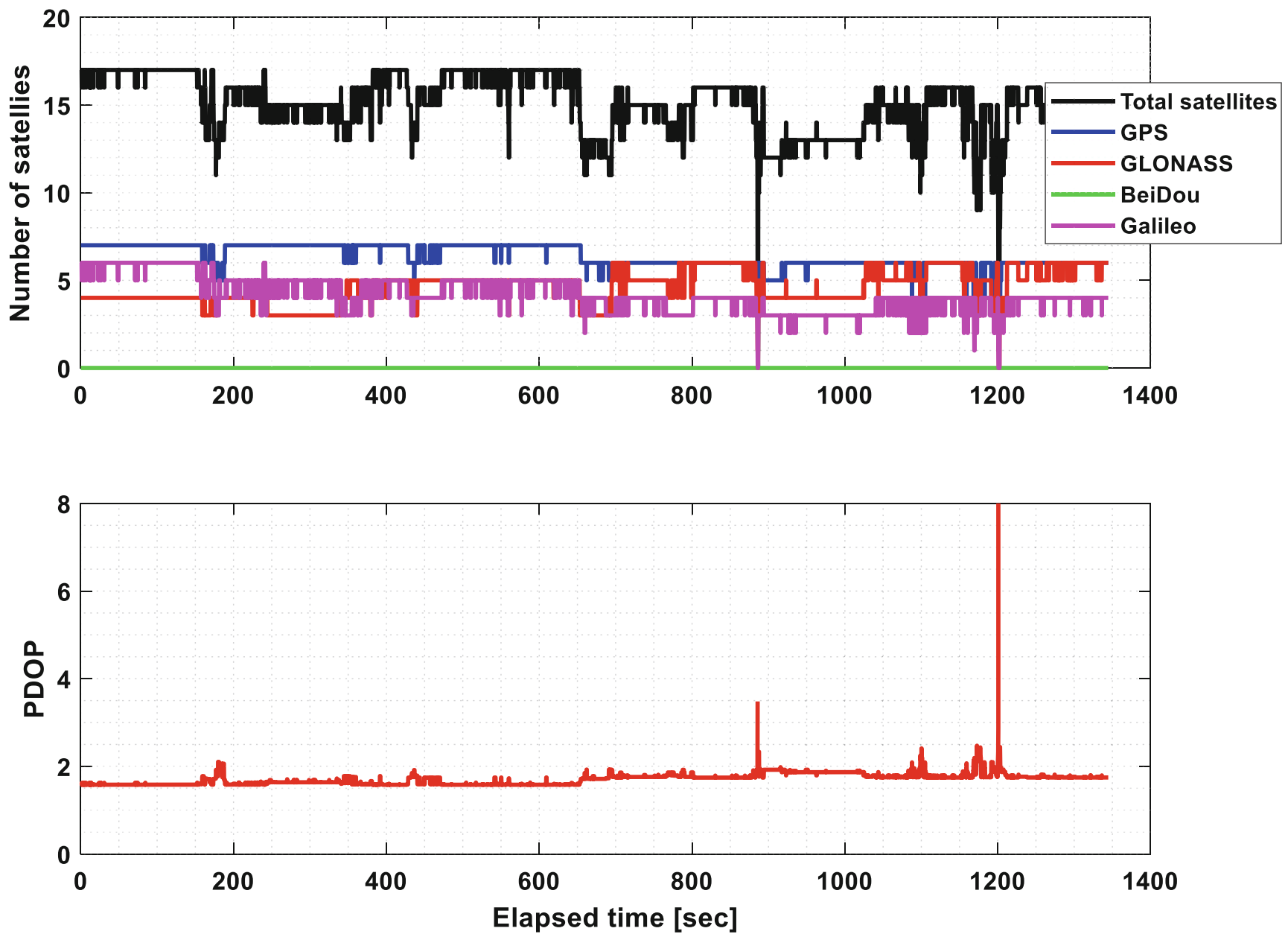

Fig. 4 Plot of available satellite and DOP vs elapsed time 


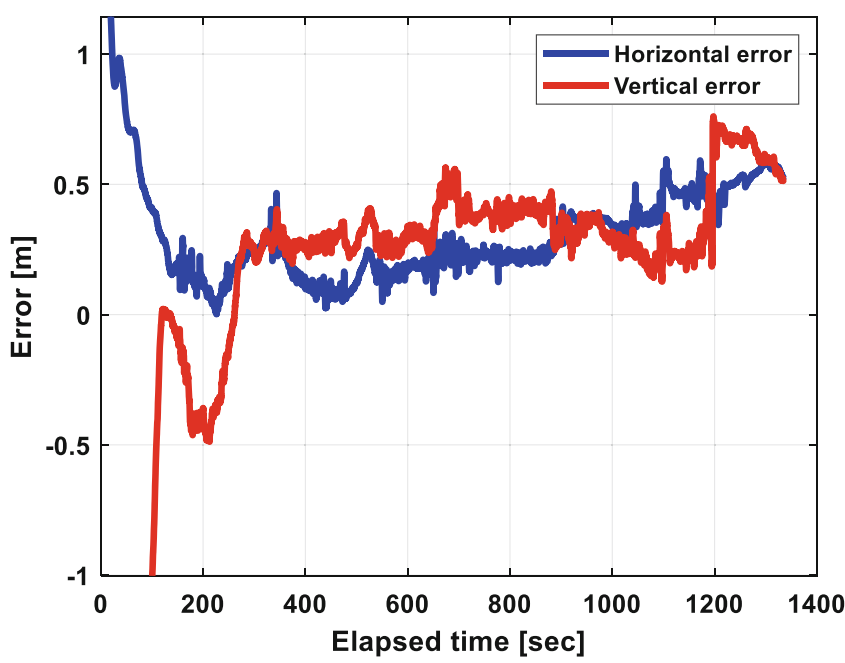

Fig. 5 Plot of horizontal and vertical error compared to the reference solution

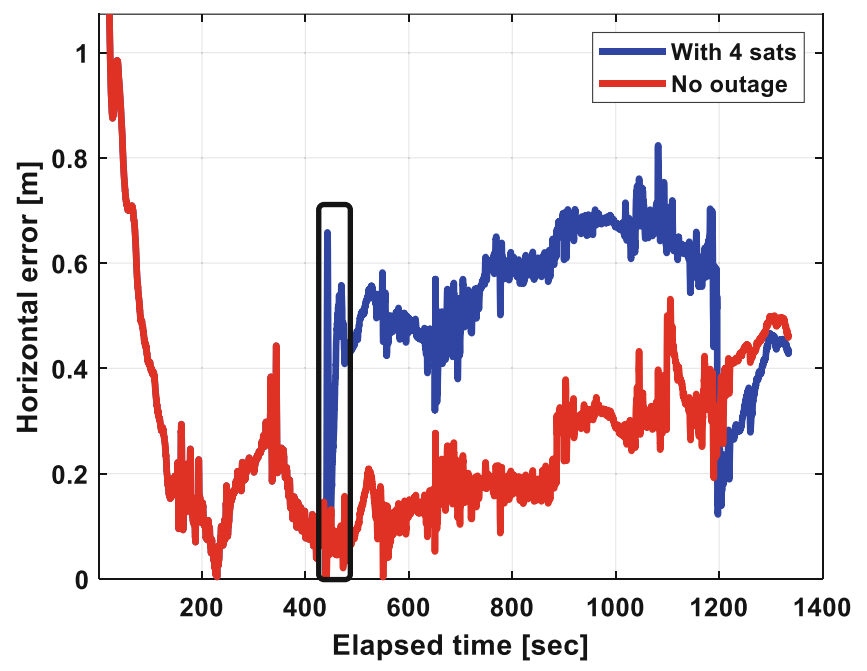

Fig. 6 Plot of horizontal error with respect to RTK Lib solution when $30 \mathrm{~s}$ outage was simulated

During the $30 \mathrm{~s}$ outage period, the solution performs with a $2 \mathrm{D} \mathrm{rms}$ of $0.4 \mathrm{~m}$ horizontally and $1.2 \mathrm{~m}$ vertically. The rms of the solution starting from the outage to end of data is $0.55 \mathrm{~m}$ horizontally and $1.1 \mathrm{~m}$ vertically. It can be noticed from Fig. 6 that the solution may not necessarily behave as it works when there is no GNSS signal outage after the outage, because every epoch of estimation process uses state estimate and covariance information from the previous epoch. The state vector and covariance information will vary based on the DOP and satellite information used in previous epoch. (Liu et al. 2018) indicated that using an SF GNSS PPP with MEMS IMU performs at a rms of $1 \mathrm{~m}$ with a $3 \mathrm{~s}$ GNSS signal outage and the accuracy was less than $10 \mathrm{~m}$ with a half minute of GNSS signal outage when there were only two satellites operating. The GNSS sensor used by (Liu et al. 2018) was Ublox EVK-M8U which has a SF GNSS chip as well a MEMS IMU in the package and global ionosphere map (GIM) products were used to reduce the ionosphere delays. A tightly-coupled algorithm using a low-cost, DF GNSS PPP with MEMS IMU performs at a less than the metre level rms error with a 30 s GNSS signal outage, which is 10 times better accuracy than a SF GNSS PPP with MEMS IMU solution.

\section{$5 \quad$ Conclusions and Future Work}

In this paper, the performance of a tightly-coupled EKF with low-cost DF GNSS PPP and MEMS IMU performance with and without outage were investigated. The algorithm performs at the decimetre-level of accuracy when there are no signal outages and it performs at the decimetre- to metrelevel accuracy during a $30 \mathrm{~s}$ outage with four satellites available. The performance of DF GNSS PPP and MEMS IMU integrated system during outage proves to be 10 times better than SF GNSS PPP with MEMS IMU. The accuracy level of the algorithm seems promising for the next generation of applications that demand higher accuracy with lower price sensors. Table 4 gives a brief summary of accuracy of the low-cost DF GNSS PPP + IMU integrated solution with and without GNSS signal outage. As part of future work, resolving ambiguities for the low-cost, DF GNSS + IMU will results in less than decimetre level accuracy and perform at an rms error of decimetre level during a half minute of GNSS signal partial absence.

Table 4 Summary of horizontal and vertical $\mathrm{rms}$ with and without signal outage

\begin{tabular}{l|l|l|l|l|l|l}
\hline \multirow{2}{*}{ Error $[\mathrm{m}]$} & During signal outage & \multicolumn{3}{l|}{ Starting from outage } & \multicolumn{2}{l}{ Overall (after convergence) } \\
\cline { 2 - 7 } & Horizontal & Vertical & Horizontal & Vertical & Horizontal & Vertical \\
\hline Without GNSS signal outage & 0.07 & 0.3 & 0.28 & 0.4 & 0.2 & 0.4 \\
\hline With 30 s GNSS signal outage (only 4 satellites available) & 0.4 & 1.2 & 0.55 & 1.1 & 0.48 & 0.9 \\
\hline
\end{tabular}


Acknowledgements The authors would like to thank Natural Sciences and Engineering Research (NSERC) and York University for financial support and German Research Centre for Geosciences (GFZ), International GNSS Services (IGS) and National Centre for Space Studies (CNES) for data provided.

\section{References}

Abd Rabbou M, El-Rabbany A (2015) Tightly coupled integration of GPS precise point positioning and MEMS-based inertial systems. GPS Solutions 19:601-609. https://doi.org/10.1007/s10291014-0415-3

Abdel-Hamid W, Abdelazim T, El-Sheimy N, Lachapelle G (2006) Improvement of MEMS-IMU/GPS performance using fuzzy modeling. GPS Solutions 10:1-11

Aggrey JE (2015) Multi-GNSS precise point positioning software architecture and analysis of GLONASS pseudorange biases. MSc Thesis, York University

Aggrey J, Bisnath S (2017) Analysis of multi-GNSS PPP initialization using dual- and triple-frequency data. In: Proceedings of the 2017 international technical meeting of the institute of navigation. Monterey, California, pp 445-458

Choy S (2018) GNSS precise point positioning. https://www.unoosa. org/documents/pdf/icg/2018/ait-gnss/16_PPP.pdf. Accessed 5 Mar 2020

Farrell J (2008) Aided navigation: GPS with high rate sensors. McGraw-Hill, Inc, New York

Groves PD (2013) Principles of GNSS, inertial, and multisensor integrated navigation systems. Artech House, Norwood

Inertial Sense (2013) Inertial Sense. In: Inertial Sense. https:// inertialsense.com/products/. Accessed 27 Sep 2019

Jekeli C (2012) Inertial navigation systems with geodetic applications. Walter de Gruyter, Berlin
Lannes A, Prieur J-L (2013) Calibration of the clock-phase biases of GNSS networks: the closure-ambiguity approach. J Geod 87:709731. https://doi.org/10.1007/s00190-013-0641-4

Liu Y, Liu F, Gao Y, Zhao L (2018) Implementation and analysis of tightly coupled Global Navigation Satellite System Precise Point Positioning/Inertial Navigation System (GNSS PPP/INS) with insufficient satellites for land vehicle navigation. Sensors 18:4305. https:// doi.org/10.3390/s18124305

Parkinson BW, Spilker JJ (1996) The global positioning system: theory and applications. American Institute of Aeronautics and Astronautics, Washington, DC

Scherzinger BM (2000) Precise robust positioning with inertial/GPS RTK. In: Proceedings of the 13th International Technical Meeting fo the Satellite Division of the Institute of Navigation (ION GPS). pp 115-162

Seepersad G (2012) Reduction of initial convergence period in GPS PPP data processing. MSc Thesis, York University

Shin E-H, Niu X, El-Sheimy N (2005) Performance comparison of the extended and the unscented Kalman filter for integrated GPS and MEMS-based inertial systems. ION NTM 2005, pp 961-969

SwiftNav (2012) Swift Navigation Precise Positioning Solutions|Piksi Multi, Duro, Duro Inertial RTK GNSS Receivers, Skylark Cloud Corrections Service, Starling Software Positioning Engine|SwiftNav. https://www.swiftnav.com/. Accessed 31 Dec 2019

Teunissen PJG, Khodabandeh A (2015) Review and principles of PPPRTK methods. J Geod 89:217-240. https://doi.org/10.1007/s00190014-0771-3

Titterton D, Weston JL, Weston J (2004) Strapdown inertial navigation technology. IET, London

Vector Nav Library I and I (2008) IMU and INS - VectorNav library. https://www.vectornav.com/support/library/imu-andins. Accessed 29 Jun 2019

Zumberge JF, Heflin MB, Jefferson DC et al (1997) Precise point positioning for the efficient and robust analysis of GPS data from large networks. J Geophys Res: Solid Earth 102:5005-5017

Open Access This chapter is licensed under the terms of the Creative Commons Attribution 4.0 International Licence (http://creativecommons. org/licenses/by/4.0/), which permits use, sharing, adaptation, distribution and reproduction in any medium or format, as long as you give appropriate credit to the original author(s) and the source, provide a link to the Creative Commons licence and indicate if changes were made.

The images or other third party material in this chapter are included in the chapter's Creative Commons licence, unless indicated otherwise in a credit line to the material. If material is not included in the chapter's Creative Commons licence and your intended use is not permitted by statutory regulation or exceeds the permitted use, you will need to obtain permission directly from the copyright holder.

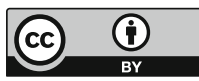

